\title{
Ythdf2-mediated $\mathrm{m}^{6} \mathrm{~A}$ mRNA clearance modulates neural development in mice
}

Miaomiao Li ${ }^{1,2 \dagger}$, Xu Zhao ${ }^{1,2^{*} \dagger}$, Wei Wang ${ }^{3}$, Hailing Shi ${ }^{4,5}$, Qingfei Pan ${ }^{6}$, Zhike Lu ${ }^{4,5}$, Sonia Peña Perez ${ }^{1}$, Rajikala Suganthan ${ }^{1}$, Chuan He $\mathrm{H}^{4,5}$, Magnar Bjørås ${ }^{1,3^{*}}$ and Arne Klungland ${ }^{1,2^{*}}$ (D)

\begin{abstract}
Background: $N^{6}$-methyladenosine $\left(m^{6} \mathrm{~A}\right)$ modification in mRNAs was recently shown to be dynamically regulated, indicating a pivotal role in multiple developmental processes. Most recently, it was shown that the Mettl3-Mettl14 writer complex of this mark is required for the temporal control of cortical neurogenesis. The $\mathrm{m}^{6} \mathrm{~A}$ reader protein Ythdf2 promotes mRNA degradation by recognizing $\mathrm{m}^{6} \mathrm{~A}$ and recruiting the mRNA decay machinery.

Results: We show that the conditional depletion of the $\mathrm{m}^{6} \mathrm{~A}$ reader protein Ythdf2 in mice causes lethality at late embryonic developmental stages, with embryos characterized by compromised neural development. We demonstrate that neural stem/progenitor cell (NSPC) self-renewal and spatiotemporal generation of neurons and other cell types are severely impacted by the loss of Ythdf2 in embryonic neocortex. Combining in vivo and in vitro assays, we show that

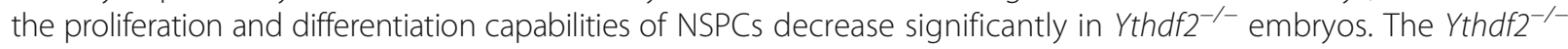
neurons are unable to produce normally functioning neurites, leading to failure in recovery upon reactive oxygen species stimulation. Consistently, expression of genes enriched in neural development pathways is significantly disturbed. Detailed analysis of the $\mathrm{m}^{6} \mathrm{~A}$-methylomes of $\mathrm{Ythdf2}^{-/-}$NSPCs identifies that the JAK-STAT cascade inhibitory genes contribute to neuroprotection and neurite outgrowths show increased expression and $\mathrm{m}^{6} \mathrm{~A}$ enrichment. In agreement with the function of Ythdf2, delayed degradation of neuron differentiation-related $\mathrm{m}^{6} \mathrm{~A}$-containing mRNAs is seen in $\mathrm{Yth}_{\mathrm{A}} \mathrm{f2^{-/- }}$ NSPCs.
\end{abstract}

Conclusions: We show that the $\mathrm{m}^{6} \mathrm{~A}$ reader protein Ythdf2 modulates neural development by promoting $\mathrm{m}^{6} \mathrm{~A}$-dependent degradation of neural development-related mRNA targets.

Keywords: Ythdf2, $N^{6}$-methyladenosine $\left(m^{6} A\right)$, Neural development, Neurogenesis, mRNA clearance

\section{Background}

Over the past decade, more than 100 post-transcriptionally modified ribonucleotides have been identified in various types of RNA [1]. Much more recently, epitranscriptomic [2] regulation at the RNA level via reversible RNA methylation has been revealed, beginning from 2011 with the discovery of the reversible potential of $N^{6}$-methyl-adenosine $\left(\mathrm{m}^{6} \mathrm{~A}\right)$ in mRNA [3]. As a post-transcriptional epitranscriptomic modification, $\mathrm{m}^{6} \mathrm{~A}$ is one of the most abundant modifications in mRNA in eukaryotes [4]. It can be written

\footnotetext{
*Correspondence: Xu.xuzha@rr-research.no; magnar.bjoras@ntnu.no; arne.klungland@medisin.uio.no

${ }^{\dagger}$ Miaomiao Li and Xu Zhao contributed equally to this work.

'Department of Microbiology, Oslo University Hospital, Rikshospitalet,

NO-0027 Oslo, Norway

Full list of author information is available at the end of the article
}

by the methyltransferase complex (Mettl3, Mettl14, Wtap, and Kiaa1429) [5], erased by demethylases (Fto and Alkbh5) $[3,6]$, and read by the binding proteins (Ythdf1-3, Ythdc1-2, and Hnrnp family proteins) [7-10].

The reversible/dynamic nature of $\mathrm{m}^{6} \mathrm{~A}$ in $\mathrm{mRNA}$ and the ability to map this modification transcriptome-wide have led to a tremendous increase in the interest and understanding of the multiple biological roles of the dynamic $\mathrm{m}^{6} \mathrm{~A}$ modification $[10,11]$. One of the evolutionarily conserved roles of the $\mathrm{m}^{6} \mathrm{~A}$ modification is the regulation of meiosis and fertility. This was shown early for the writers of $\mathrm{m}^{6} \mathrm{~A}$ in model organisms [12] and also for the mammalian $\mathrm{m}^{6} \mathrm{~A}$ eraser Alkbh5 [6] and the $\mathrm{m}^{6} \mathrm{~A}$ reader protein Ythdf2 [13]. The depletion of the $\mathrm{m}^{6} \mathrm{~A}$ eraser Fto in mammalian cells causes defects in

(C) The Author(s). 2018 Open Access This article is distributed under the terms of the Creative Commons Attribution 4.0 International License (http://creativecommons.org/licenses/by/4.0/), which permits unrestricted use, distribution, and 
energy homeostasis and adipocyte differentiation [14]. It is worth mentioning that a loss-of-function mutation in the Fto gene causes growth retardation and multiple malformations in humans [15]. The writer Mettl3 is crucial for maintaining mouse stem cell pluripotency, regulating the reprogramming of somatic cells and the circadian rhythm, and targeting of the gene in mouse causes early embryonic lethality [16-20]. The most recent studies in hematopoietic stem/progenitor cells have uncovered the crucial role of Mettl3 in determining cell fates during vertebrate embryogenesis $[21,22]$. The $\mathrm{m}^{6} \mathrm{~A}$ reader proteins Ythdf1-3 share a set of common mRNA targets and spatiotemporal interplay with each other cooperatively control translation and decay of these common targets in the cytosol [23]. The $\mathrm{m}^{6} \mathrm{~A}$ readers Ythdc1 and Hnrnpa2b1 regulate splicing and processing of their mRNA targets [8, 24], while Ythdc2 affects translation efficiency as well as stability of target mRNAs [9].

Recently, mutant models of the mammalian $\mathrm{m}^{6} \mathrm{~A}$ readers reveal interesting phenotypes, which again include spermatogenesis [9] and oocyte competence [25]. Moreover, Ythdf2-dependent, $\mathrm{m}^{6} \mathrm{~A}$-modified mRNA clearance was shown to impact the highly regulated maternal-to-zygotic transition (MZT) in zebrafish [7, 13]. In Drosophila, $\mathrm{m}^{6} \mathrm{~A}$ writer (Ime4, dMettl14) and reader (Yt521-b) mutants exhibit flight defects and poor locomotion due to impaired neuronal functions [26]. Most recently, the $\mathrm{m}^{6} \mathrm{~A}$ writer Mettl14 was shown to be required for the temporal control of mammalian cortical neurogenesis [27]. These findings strongly suggest the potential role of $\mathrm{m}^{6} \mathrm{~A}$ modification during nervous system development, which might be conserved across species. Many histone and DNA encoded epigenetic mechanisms are uncovered to be conserved in this process. Thus, addressing the role of $\mathrm{m}^{6} \mathrm{~A}$ methylation in mRNA will be an exciting new field to explore and will shed new light on neural development.

The $\mathrm{m}^{6} \mathrm{~A}$ reader Ythdf 2 is essential for oocyte competence and mutation of it causes female infertility [25]. Here we describe the early brain developmental failure of mice lacking Ythdf2 due to failure to regulate neural stem/progenitor cell (NSPC) proliferation and differentiation. During embryonic development, apical progenitor cells in the ventricular zone (VZ) serve as primitive neural stem cells that give rise to both the neuronal and glial lineages directly or produce secondary progenitors, termed the basal progenitor, in the subventricular zone (SVZ) in a precisely regulated spatiotemporal order [28]. In this study, Ythdf2 knockout embryos displayed delayed cortical neurogenesis. In vivo and in vitro experiments proved that Ythdf2-deficient NSPCs display decreased proliferation rates. Furthermore, Ythdf2-deficient NSPCs could naturally differentiate to neurons but not glial cells in vitro. However, the properties of differentiated neurons were influenced, seen as less neurite outgrowth and shorter neurites. Removal of Ythdf2 increased the sensitivity of neurons to reactive oxygen species (ROS) stress and decreased their recovery capability. RNA-seq combined with $\mathrm{m}^{6} \mathrm{~A}$-seq uncovered that the $\mathrm{m}^{6} \mathrm{~A}$ modified mRNAs involved in negative regulation of neural development were up-regulated in Ythdf2-deficient NSPCs, in agreement with the function of Ythdf2. The $\mathrm{m}^{6} \mathrm{~A}$-modified mRNA targets, recognized by the Ythdf 2 protein in the wild type, were characterized by delayed degradation in Ythdf2 knockout embryos. Taken together, our findings reveal the critical functions of $\mathrm{m}^{6} \mathrm{~A}$ modification and its binding protein Ythdf2 in neural development.

\section{Results and discussion}

\section{Ythdf $2^{-/-}$targeted mice are embryonic lethal}

In order to study the biological function of the $\mathrm{m}^{6} \mathrm{~A}$ reader Ythdf2, we generated conditional C57BL/6 Ythdf2 targeted mice with LoxP sites flanking the $5^{\prime}$ UTR and exon 1 of the endogenous Ythdf2 locus using CRISPR-Cas9 technology (Fig. 1a). The Ythdf2 $2^{+/ l o x p}$ mice were crossed with mice ubiquitously expressing Cre-recombinase to generate the Ythdf $2^{+/-}$mice. Then to get Ythdf $2^{-/-}$mice, we intercrossed heterozygous $Y$ thd $f 2^{+/-}$mice. Interestingly, no viable $Y$ thd $f 2^{-/-}$newborn mice were identified in this particular knockout strain. The ratio of wild-type, hetero-, and homozygous knockout mice was not consistent with the expected 1:2:1 Mendelian ratio. Noteworthy, the number of postnatal Ythdf $2^{+/-}$mice indicated semi-lethality for these mice (Fig. 1b). Furthermore, 34\% of Ythdf $2^{+/-}$ surviving mice have malfunctioning eyes, with eyelids remaining closed (Additional file 1: Figure S1b). Many factors might contribute to this [29, 30], such as dysfunction of hypothalamic nerve control, but this was not studied further here.

To assess the stage of developmental failure, we collected embryos at E12.5 and E14.5 from heterozygote intercrosses and genotyped them by both PCR with primers flanking and inside the deleted genomic region (Fig. 1a, c) and western blotting with Ythdf2 antibody (Fig. 1d). PCR and western blot analysis confirmed that the expression of Ythdf2 is completely depleted in Ythdf $2^{-/-}$embryos. The Mendelian distribution of wild type, Ythdf $2^{+/-}$, and Ythdf $2^{-/-}$was 1:2:1 when genotyped at embryonic stages E12.5-14.5 (Fig. 1b), suggesting the stage of embryonic lethality after E14.5. Therefore, we isolated embryos at E18.5 for further analysis. At this stage, 3 out of 41 embryos were genotyped as $Y$ thdf $2^{-/-}$ (data not shown). Despite the genotype ratio being normal at E12.5 and E14.5, the average number of embryos per litter was significantly less in $Y$ thdf $2^{+/-}$ intercrosses compared with wild-type intercrosses, 




Fig. 1 Ythdf $2^{-/-}$mice are embryonic lethal. a The gene-targeting strategy to disrupt the Ythdf2 gene in mouse. Conditional Ythdf2 gene-targeted mouse contains LoxP sites flanking the $5^{\prime}$ UTR and exon 1 of the endogenous Ythdf2 locus. WT_F wild-type forward primer, WT_R wild-


Ythdf2 $^{+/-}$intercrosses. The number and genotype of embryos at E12.5/E14.5 and postnatal are indicated. c PCR analysis of embryo tail DNA showing a 271-bp wild-type band (WT) and a 550-bp targeted band (KO) with primers displayed in a. $\mathbf{d}$ Western blot analysis of the Ythdf2 expression in wild-type and Ythdf2 $2^{-/-}$embryos. Two samples for each genotype. Actin was used as loading control. e Numbers of embryos per litter at E12.5/E14.5 and E18.5 from wild-type or heterozygous intercrosses. Error bars represent mean \pm standard deviation, $n=7$ litters. ${ }^{*} P<0.05,{ }^{* *} P<0.01,{ }^{* * *} P<0.001$, Student's $t$-test

especially at the late embryonic stage E18.5 (Fig. 1e). It was reported that removal of Ythdf2 in zebrafish leads to $31.3 \%$ cell arrest and lethality at the one-cell stage by $Y$ thdf2 $2^{+-}$intercross matings [13], consistent with our finding of $30 \%$ less embryos at E12.5 and
E14.5. According to our data, the major lethality of Ythdf $2^{-/-}$embryos occurred between E14.5 and E18.5. Therefore, disruption of the Ythdf2 gene results in embryonic lethality during the late developmental stages of embryogenesis. 
Ythdf $^{-/-}$mice display abnormal cortical development To determine how depletion of Ythdf2 affects embryonic development, we dissected embryos at E12.5, E14.5, and E18.5. Although Ythdf2 $2^{-/}$embryos at E12. 5 and E14.5 were alive and appeared normal, sagittal sectionings of the whole embryos and H\&E staining uncovered dramatically decreased overall cortical thickness of Ythdf $2^{-/-}$embryonic fore brains (Fig. 2a). Compared with their wild-type littermates, there was a general $56 \mu \mathrm{m}$ decrease in the cortical layer at E12.5 and $40 \mu \mathrm{m}$ decrease in the cortical layer at E14.5, yet the cortexes of both genotypes grew from E12.5 to E14.5 (Fig. 2b).
The Ythdf2 $2^{+/-}$mice are semi-lethal. Thus, we also analyzed a cohort of Ythdf2 $2^{+-}$mice and found a mean $29 \mu \mathrm{m}$ decrease in the cortical layer at E12.5 and a mean $24 \mu \mathrm{m}$ decrease in the cortical layer at E14.5 (Fig. 2a, b). We suspected that the delayed cortical development derived from a defect in the early stages of neurogenesis. In order to determine whether Ythdf2 expression is temporally associated with brain development, we analyzed the expression of Ythdf2 in brain samples by quantitative RTPCR at E12.5, E13.5, E17.5, and E18.5. Ythdf2 was highly expressed during the early stage of neural development (Additional file 1: Figure S1a).



C

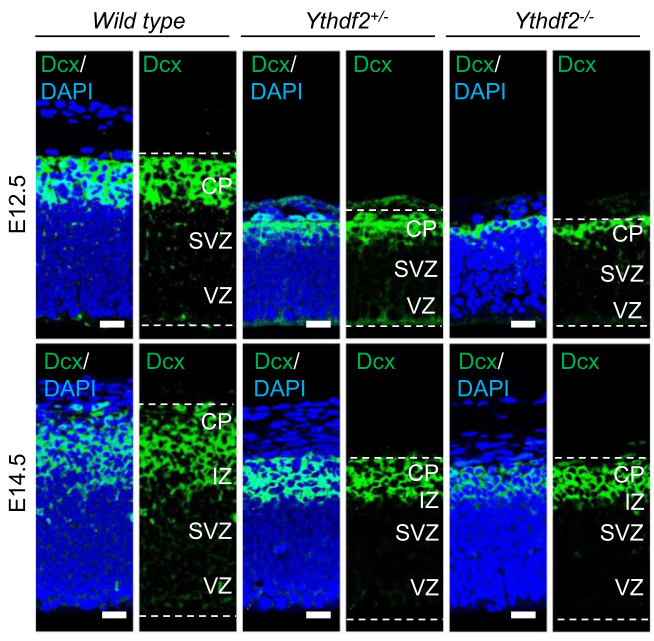

b

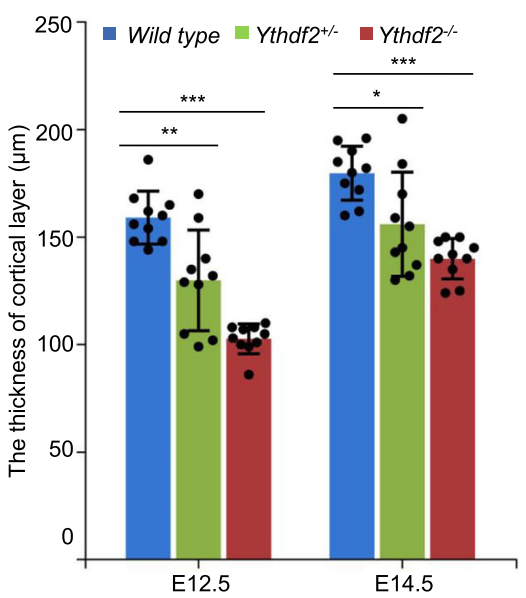

d

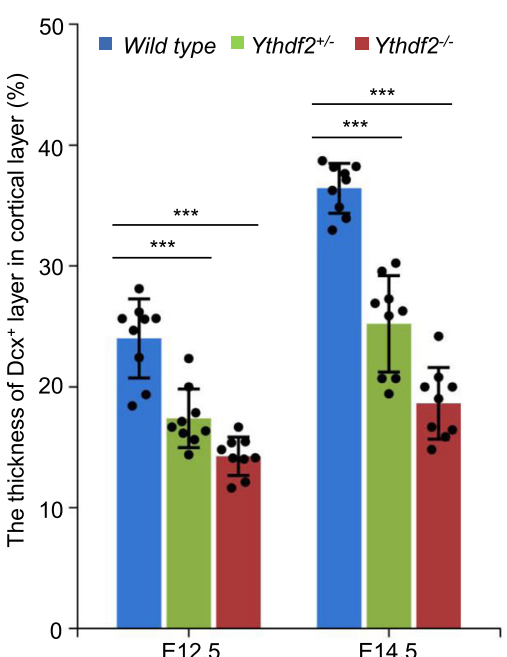

Fig. 2 Ythdf2 is required for normal embryonic cortical development. a Sagittal brain sections of E12.5 and E14.5 were stained with H\&E. An enlarged

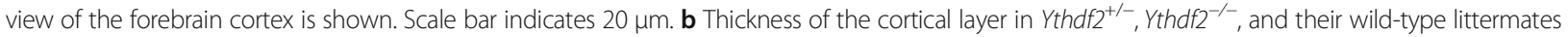
at E12.5 and E14.5. Error bars represent mean \pm standard deviation, $n=3$ embryos and 3 technical replicates. c Immunostaining of E12.5 and E14.5 brain

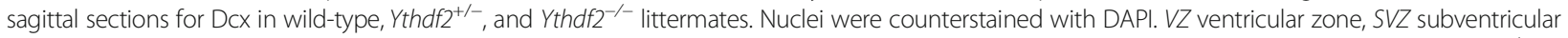
zone, IZ intermediate zone, CP cortical plate. $\mathbf{d}$ Ratio of the thickness of Dcx-immunolabeled neuronal layers over cortical layers in Ythdf2 ${ }^{+/-}$ and $Y$ thdf $2^{-1-}$ compared with wild type. Error bars represent mean \pm standard deviation, $n=3$ embryos and 3 technical replicates. ${ }^{*} P<0.05$, ${ }^{*} P<0.01,{ }^{* *} P<0.001$, Student's t-test 
To further define the neuronal developmental failure associated with Ythdf2 deficiencies, embryonic brain slices at different developmental stages were stained with the immature neuron marker doublecortin (Dcx). At E12.5, the neuronal layer of Ythdf2 $2^{-/-}$and Ythdf2 $2^{+-}$ embryos was significantly thinner than that of the wild type, here shown as the ratio of the thickness of Dcximmunolabeled neuronal layers over cortical layers (Fig. 2c, d). Taken together, the in vivo evidence indicates a striking phenotype of retarded cortical development, resulting from decreased neurogenesis at the early stages of embryonic brain development.

Basal progenitor cells are decreased in Ythdf2 ${ }^{-/-}$embryos Neural stem/progenitor cells (NSPCs) and immature neurons are the major cortical components at E12.5 and E14.5 in mice. NSPCs give rise to neurons. Given the profound effects of $Y$ thdf 2 targeting on embryonic brain development, we examined the proliferation and differentiation capability of NSPCs during development. The T-box transcription factor Eomes (Tbr2) is specifically expressed in basal progenitor cells, predominantly in the SVZ, which primarily differentiate into superficial layer neurons. In E12.5 and E14.5 Ythdf2 ${ }^{-/-}$embryos and, to a lesser extent, Ythdf2 $2^{+/-}$embryos, there was a dramatic loss of basal progenitor cells, displayed by the obviously thinner Tbr2 layer, compared to wild type littermate embryos (Fig. 3a). The sex determining region Y-box2 (Sox2) is a marker for apical progenitor cells located in the VZ, which can produce deep layer neurons and basal progenitor cells [31]. The ratio of Tbr2-positive cells to total progenitors $\left(\mathrm{Tbr}^{+} / \mathrm{Sox}^{+}\right)$was decreased markedly at E12.5 and E14.5 in Ythdf2 $2^{-/-}$and Ythdf2 $2^{+/-}$embryos compared with the wild types (Fig. 3b), suggesting the decrease in neurons $\left(\mathrm{Dcx}^{+}\right)$associates with a reduction in the basal progenitor population in SVZ. However, there was no obvious difference in Sox2-positive apical progenitor cells in VZ layer (Fig. 3a).

\section{Mitotic capability of apical progenitor cells is impaired in Ythdf2 $^{-/-}$embryos}

The non-self-renewing basal progenitors only experience one or two mitotic cycles, and the majority of basal progenitors are established by asymmetric division of apical progenitor cells during early cortical development [32, 33]. We propose that the decrease in basal progenitors $\left(\mathrm{Tbr}^{+}\right)$might be caused by the reduced mitotic capability of the Ythdf2-depleted apical progenitor cells. The E12.5 and E14.5 sagittal sections of wild type, Ythdf $2^{+/-}$, and Ythdf $2^{-/-}$embryos were co-stained with the mitotic phase marker phospho-histone $\mathrm{H} 3$ (Phh3) and Sox 2 to quantify the mitotic capability of the apical progenitor cells. The number of Phh3-positive cells de-



compared with wild type at E12.5 and E14.5. In Ythdf2 $2^{+/-}$ cortex, the number of Phh3-positive cells was significantly reduced compared to the wild type cortex, yet was higher than in Ythdf $2^{-/-}$cortex (Fig. 3c, d). Additionally, apical progenitor cells could also maintain the population by several rounds of symmetric division in the VZ layer [34]. As there were no obvious changes in the number of apical progenitor cells $\left(\mathrm{Sox}^{+}\right)$in the VZ layer, we concluded that Ythdf2-dependent defective neurogenesis was caused by the decreased generation of basal progenitors from apical progenitors.

\section{Ythdf2 ${ }^{-/-}$NSPCs exhibit decreased proliferation in vitro}

To further understand how Ythdf2 regulates neurogenesis, we cultured neurospheres consisting of NSPCs derived from E14.5 wild-type and Ythdf2 ${ }^{-/-}$embryonic fore brain. The Ythdf $2^{-/-}$neurospheres were smaller than the wild-type spheres (Additional file 1: Figure S2a, b). We first monitored the influence of Ythdf2 on NSPC proliferation. NSPCs dissociated from the primary neurospheres were seeded for proliferation testing and the cell growth was determined at 0, 24, 72, and $120 \mathrm{~h}$. Compared with the wild type, Ythdf2 ${ }^{-/}$NSPCs showed a slightly decreased proliferation rate after $24 \mathrm{~h}$ and a more pronounced reduction after $72 \mathrm{~h}$ culturing (Fig. 4a). This result is in agreement with the decreased mitotic capability of stem/progenitor cells observed in vivo.

\section{Ythdf2-deficient NSPCs show impaired neural differentiation}

In differentiation assays, NSPCs dissociated from neurospheres produce both neurons and glial cells after 5 days culturing. We first assessed the mRNA expression profile of Ythdf2 in wild-type neurospheres during differentiation by RT-qPCR. The expression of Ythdf2 was upregulated from Day 0 (D0) to D3 during differentiation and remained high till D5, suggesting the involvement of Ythdf2 in regulating differentiation (Fig. 4b). Neuronal and glial cell lineages can be identified by staining with antibody against microtubule associated protein 2 (Map2) or glial fibrillary acidic protein (Gfap), respectively. We quantified the percentages of Gfap-positive cells for Ythdf2 $2^{-/-}$and wild-type at D5 and D7. Dramatic reduction of glial cells, with abnormal branches $\left(\mathrm{Gfap}^{+}\right)$, was observed in differentiated Ythdf2 ${ }^{-/-}$neurospheres (Fig. 4c, d). However, we did not observe a significant different ratio of Ythdf $2^{-/-}$ neurons $\left(\mathrm{Map2}^{+}\right)$at D5, while the ratio of Ythdf2 $2^{-/}$ neurons declined significantly more than the wild type at D7. These results were further substantiated by neuron progenitor antibody neuron-specific class III beta-tubulin (Tuj1) and glial progenitor antibody S100 calcium-binding protein B $(S 100-\beta)$ staining at D3 and D5 (Additional file 1: Figure S2c, d). At D3, the number of glial lineage 
a



C



b

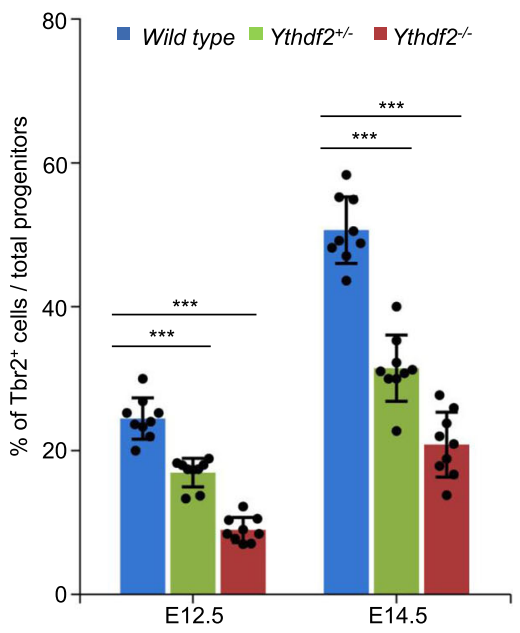

d

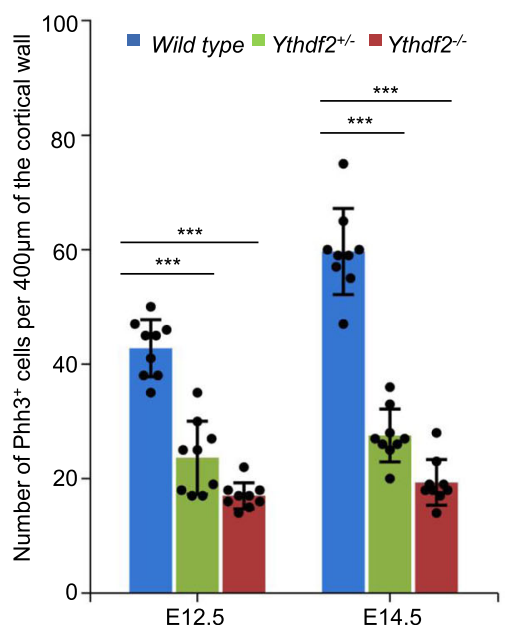

Fig. 3 The number of basal progenitors and mitotic capability of apical progenitors depends on Ythdf2. a Immunostaining of E12.5 and E14.5

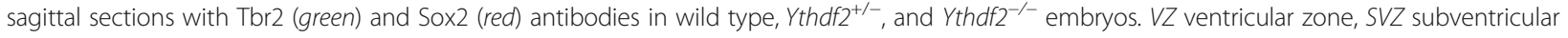
zone, IZ intermediate zone, CP cortical plate. Nuclei were counterstained with DAPI. b Percentage of Tbr2 ${ }^{+}$cells over Tbr2 $/$Sox $2^{+}$at E12.5 and E14.5. Error bars represent mean \pm standard deviation, $n=3$ biological and 3 technical replicates. Scale bars, $20 \mu m$. c Immunostaining of E12.5 and E14.5 sagittal sections with Phh3 (green) and Sox2 (red) antibodies in wild type, Ythdf2 ${ }^{+/}$, and Ythdf2 $^{-/-}$embryos. Nuclei were counterstained with DAPI. d Number of Phh3 ${ }^{+}$cells per $400 \mu \mathrm{m}$ of the cortical wall at E12.5/E14.5 from c. Error bars represent mean \pm standard deviation, $n=3$ biological and 3 technical replicates. ${ }^{*} P<0.05,{ }^{* *} P<0.01,{ }^{* *} P<0.001$, Student's $t$-test. Scale bars, $20 \mu \mathrm{m}$

progenitors had already declined in $Y$ thdf $2^{-/-}$cells, while no difference was observed for neuronal lineage progenitor cells (Additional file 1: Figure S2c, d). The TUNEL assay showed significantly more dead $Y t h d f 2^{-/-}$ cells, which might result from impaired differentiation (Additional file 1: Figure S2e, f).

\section{Ythdf2-deficient neurons display abnormal neurite outgrowth and increased sensitivity to arsenite}

Whereas neuronal lineage differentiation Map2 $^{+}$or Tuj $1^{+}$) was not affected at D5 in Ythdf2 ${ }^{-/-}$cells, the morphological analysis of Map2-positive cells showed that $Y$ thdf $2^{-/-}$differentiated neurons had less and shorter primary neurites (axons and dendrites). The mean number of branching neurites per neuron in Ythdf $2^{-/-}$differentiated cells is less than in the wild type (Fig. 4e), and the mean length of the longest neurite in Ythdf $2^{-/-}$differentiated cells is shorter than in the wild type (Fig. 4f). The neurite outgrowth is pivotal in neuronal development and maturation, synaptic formation, neuronal function, and functional recovery in diseases [35]. The severe effect on neurite branching and extension of 


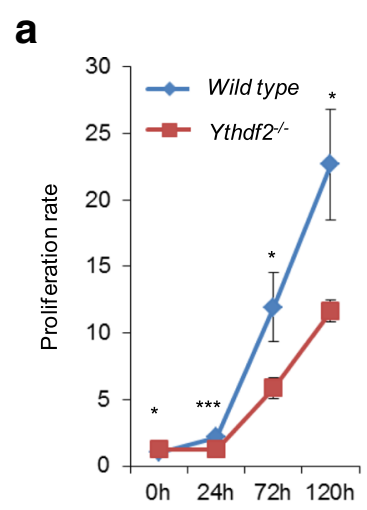

b

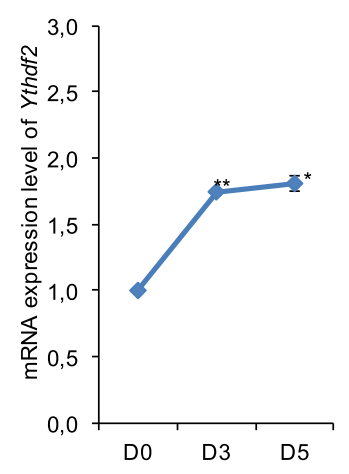

e

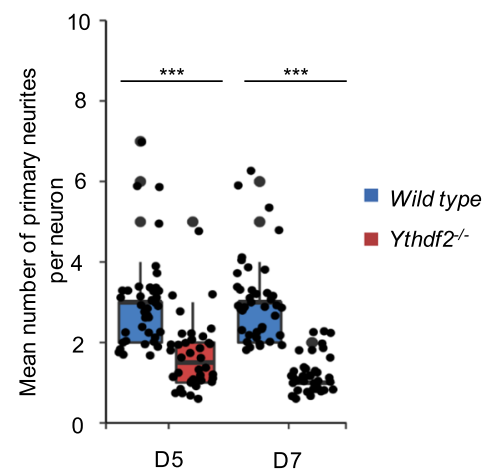

d
D5
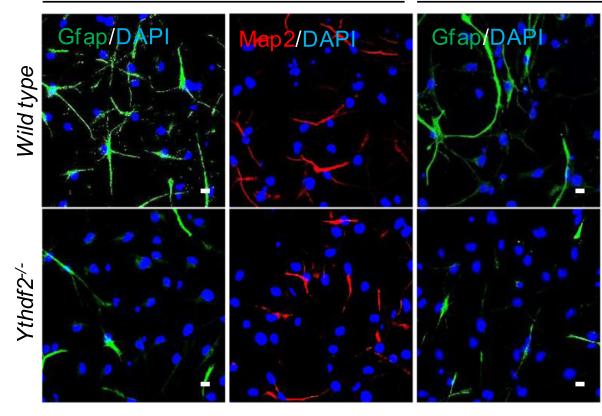

D7
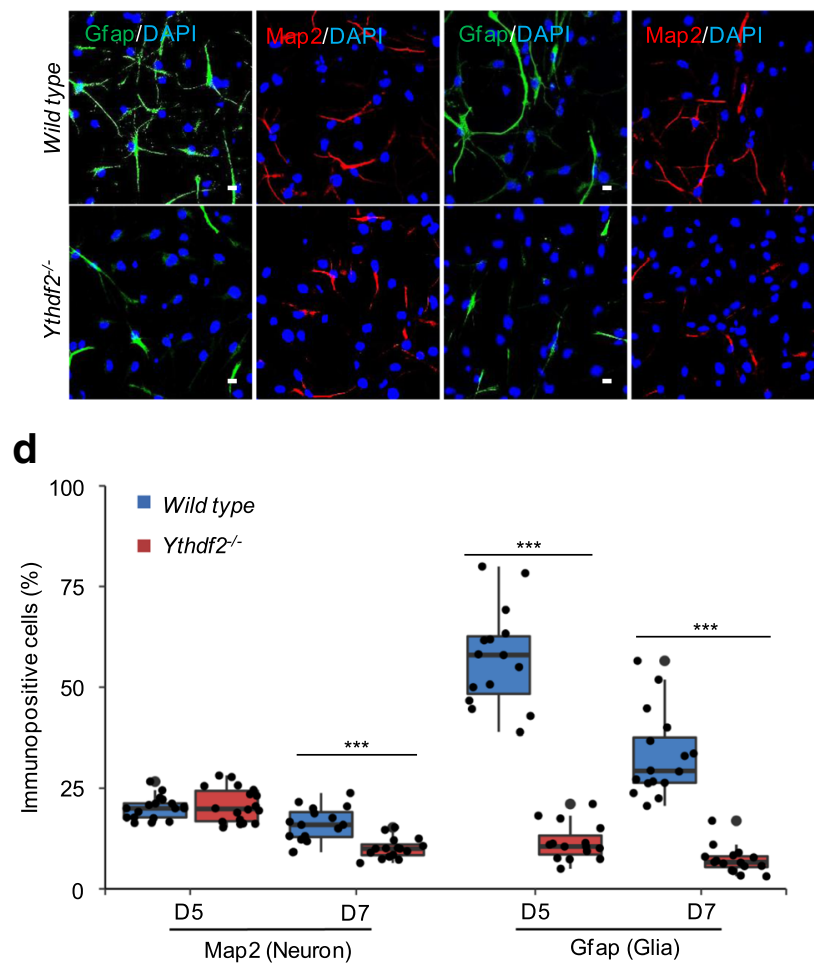

f

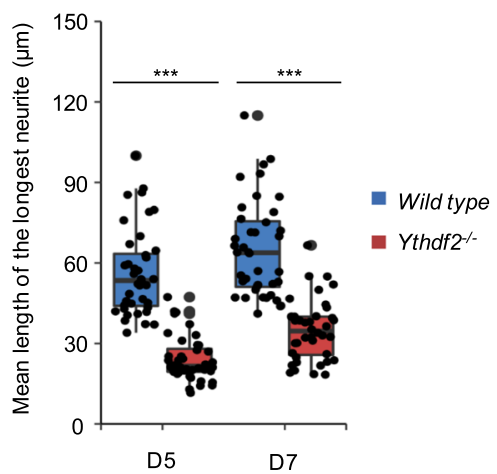

Fig. 4 Ythdf2 ${ }^{-1-}$ NSPCS exhibit decreased proliferation and defects in natural differentiation in vitro. a Number of viable NSPCs at 0, 24, 72, and $120 \mathrm{~h}$ monitored by signal intensity of Presto Blue reagent. Proliferation rate was calculated by normalizing to wild type at $0 \mathrm{~h}$. $\mathbf{b} \mathrm{mRNA}$ expression levels of Ythdf2 during NSPC differentiation. Cells were collected at differentiation Day 0 (D0), 3, and 5. Isolated total RNAs were applied for RT-qPCR analysis. Actin was used as normalization control. c Immunostaining of Map2 ${ }^{+}$and Gfap $^{+}$cells differentiated from E14.5 neurospheres at D5 and D7. Nuclei were counterstained with DAPI. Scale bar indicates $20 \mu \mathrm{m}$. $\mathbf{d}$ Percentage of Map2 or Gfap positive cells. Error bars represent mean \pm standard deviation, $n=3$ biological repeats and 3 technical replicates. e Mean number of primary neurites per neuron $\left(\mathrm{Map2}^{+}\right) \cdot n=20$ neurons for each biological repeat. $\mathbf{f}$ Mean length of the longest neurite of neurons $\left(\mathrm{Map2}^{+}\right) \cdot n=20$ neurons for each biological repeat. ${ }^{*} P<0.05,{ }^{*} P<0.01,{ }^{* * *} P<0.001$, Student's $t$-test

Ythdf2 $2^{-/-}$neurons might also contribute to the defective neurogenesis during neural development.

Besides, we found that differentiated neurons in vitro were more sensitive to arsenite treatment. Arsenite was demonstrated to induce oxidative stress by generating ROS and depleting antioxidants in cell lines and mammalian brain $[36,37]$. It is reported that after arsenite treatment, Ythdf2 can co-localize with $\mathrm{P}$ body to regulate mRNA decay [7]. We treated differentiated neurons with $5 \mu \mathrm{M}$ arsenite for $24 \mathrm{~h}$ in vitro, followed by recovery in fresh medium for $24 \mathrm{~h}$ (Additional file 1 : Figure S3a). For wild-type neurons, the mean length of 
neurites was shortened and the number of neurites reduced after 24-h arsenite exposure (Additional file 1: Figure S3b, c). However, after 24-h culture in fresh medium, the remaining neurites recovered to the original length and the neurite number partially increased as the growth of new neurites needs longer time (Additional file 1: Figure S3b, c). In contrast, Ythdf $2^{-/-}$neurons showed increased sensitivity to arsenite exposure compared to wild-type neurons. After 24-h recovery, Ythdf $2^{-/-}$neurites could not outgrow to the original length and no new neurites projected.

\section{Negative regulation of neural development pathways enriched in Ythdf $2^{-/-}$neurospheres}

To address the molecular mechanism of modulating NSPC proliferation and differentiation, we performed mRNA sequencing in the wild-type and Ythdf2 $2^{-/-}$ neurospheres with three biological replicates. We identified 2144 up-regulated differentially expressed genes (DEGs) and 1756 down-regulated DEGs (Additional file 1: Figure S4a). With more stringent criteria (fold change $>1.5, P<0.05$ in three replicates), 151 significantly up-regulated and 316 significantly down-regulated


Interestingly, the up-regulated genes were significantly associated with axon guidance, synapse assembly, neuron differentiation, and apoptosis. All these biological processes are subordinate to nerve development (Additional file 1: Figure S4b). The JAK-STAT signaling pathway is up-regulated in neurons and glial cells, which contributes to the neuroprotection and neurite outgrowth [38, 39]. The genes, highly enriched for Gene Ontology (GO) term "negative regulation of JAK-STAT cascade", inhibit this cascade, such as Flrt2, Flrt3, Ptprd, and Lrrtm1 and 4. On the contrary, clustered terms, such as "positive regulation of cell differentiation", "positive regulation of transcription", "positive regulation of GTPase activity", and "negative regulation of neuron apoptotic process", were dominant in down-regulated genes.

$\mathrm{m}^{6} \mathrm{~A}$-methylomes in wild-type and $\mathrm{Ythdf2}^{-/-}$neurospheres In order to gain more insight about the role of $Y$ thdf $2^{-/-}$in $\mathrm{m}^{6} \mathrm{~A}$ mRNA decay, we compared the $\mathrm{m}^{6} \mathrm{~A}$ methylome of wild-type and $Y$ thdf2 $2^{-/-}$neurospheres. Initially, we quantified the $\mathrm{m}^{6} \mathrm{~A} / \mathrm{A}$ ratio of the total mRNAs purified from the wild-type and Ythdf $2^{-/-}$ neurospheres by LC-MS/MS. In Ythdf2 ${ }^{-/-}$neurospheres, the $\mathrm{m}^{6} \mathrm{~A}$ abundance was increased by around $10 \%$ on average compared with the wild type (Fig. 5a). This is consistent with the $\mathrm{m}^{6} \mathrm{~A}$-dependent RNA decay function of Ythdf2 [7] and correlates very well with a study in zebrafish on the role of Ythdf2 in the maternal-tozygotic transition [23]. We identified 16,626 common $\mathrm{m}^{6} \mathrm{~A}$ sites from 8201 genes and 17,734 common $\mathrm{m}^{6} \mathrm{~A}$ sites from 8585 genes in three biological replicates of wild-type and $Y$ thdf $2^{-/-}$neurospheres, respectively (Additional file 1: Figure S5a). The highly overrepresented $\mathrm{m}^{6} \mathrm{~A}$ RRACH $(\mathrm{R}=\mathrm{G} / \mathrm{A}, \mathrm{H}=\mathrm{U} / \mathrm{A})$ motif identified using the HOMER algorithm in both wild-type $(P=1 \mathrm{e}-471)$ and $Y$ thdf $2^{-/-}(P=1 \mathrm{e}-475)$ neurospheres proved the successful enrichment of $\mathrm{m}^{6} \mathrm{~A}$-modified mRNA (Fig. 5b and Additional file 1: Figure S5b). The $\mathrm{m}^{6} \mathrm{~A}$ sites were significantly enriched at start codons, stop codons, and 3' UTRs. The $\mathrm{m}^{6} \mathrm{~A}$ profile is thus in very good agreement with those reported previously (Fig. 5c and Additional file 1: Figure S6a).

Based on the statistics from three biological replicates, $3095 \mathrm{~m}^{6} \mathrm{~A}$ sites from 2464 genes and $4109 \mathrm{~m}^{6} \mathrm{~A}$ sites from 2619 genes were identified to have lower or higher $\mathrm{m}^{6} \mathrm{~A}$ levels in three biological replicates of Ythdf2 $2^{-/-}$ neurospheres (Fig. 5d and Additional file 1: Figure S6b). $m^{6} \mathrm{~A}$ sites with significantly higher enrichment (fold change >1.5) in all three Ythdf2 ${ }^{-/-}$replicates were analyzed further. Based on this stringent criterion, 78 $\mathrm{m}^{6} \mathrm{~A}$ sites from 69 genes were markedly up-regulated. These genes were enriched for functional clusters like transcription regulation, phosphorylation, and neuron projection development (Additional file 1: Figure S7a). On the other hand, $102 \mathrm{~m}^{6} \mathrm{~A}$ sites from 99 genes were down-regulated. These genes were enriched for functional clusters like transcription regulation, transport, rhythmic process, and apoptosis (Additional file 1: Figure S7b). Among these 168 genes, 115 genes had conserved $\mathrm{m}^{6} \mathrm{~A}$ sites across samples, while 65 and 54 genes had newly occurring or absent $\mathrm{m}^{6} \mathrm{~A}$ sites, respectively, in all three Ythdf $2^{-/-}$neurospheres (Additional file 1: Figure S7c).

\section{Ythdf2 is required for degradation of genes related to neuron differentiation}

It is well established that Ythdf2 specifically binds mRNAs containing $\mathrm{m}^{6} \mathrm{~A}$ and promotes mRNA decay $[1,7]$. In Ythdf2-depleted zebrafish embryos, Ythdf2-targeted mRNAs had extended lifetimes as seen by increased mRNA levels [13]. Hence, we focused on verifying candidate genes with increased mRNA transcripts and enrichment of $\mathrm{m}^{6} \mathrm{~A}$ sites. Among these genes, Nrp2 and Nrxn3 were involved in nerve development and cell differentiation; Flrt2 and Ptprd were enriched in negative regulation of JAK-STAT cascade, regulation of synapse assembly and axon guidance, and neuron differentiation; $D d r 2$ was related to fibroblast proliferation; Hlf was involved in rhythmic process; and Nrp2 and other genes showed enrichment of representative $\mathrm{m}^{6} \mathrm{~A}$ peaks in Ythdf2 $2^{-/}$neurospheres (Fig. 5e and Additional file 1: Figure S8). To further substantiate these findings, we performed $\mathrm{m}^{6} \mathrm{~A}$ immunoprecipitation (IP) combined with RT-qPCR. Consistent with our initial findings, $\mathrm{m}^{6} \mathrm{~A}$ IP showed that $\mathrm{m}^{6} \mathrm{~A}$ levels increased significantly, while 

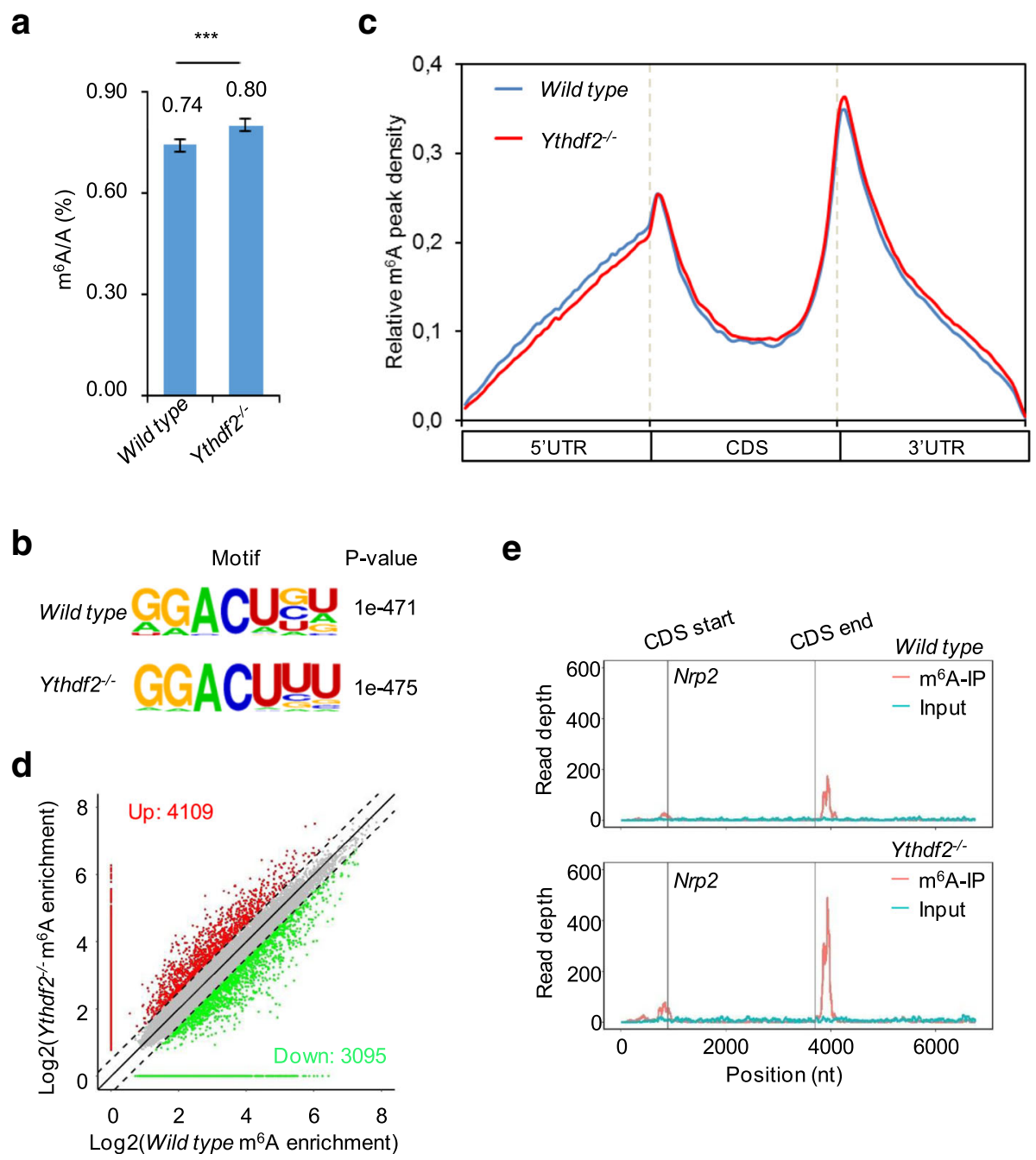

Fig. 5 Overview of $\mathrm{m}^{6} \mathrm{~A}$ methylomes in wild-type and $Y$ thdf2 ${ }^{-/-}$neurospheres. a The $\mathrm{m}^{6} \mathrm{~A}$ contents of mRNAs isolated from wild type and $Y$ thdf2 ${ }^{-/-}$were quantified by LC-MS/MS. b Sequencing motif in $\mathrm{m}^{6} \mathrm{~A}$ peaks verified in wild type and $Y$ thdf $2^{-1-}$ with HOMER database. $\mathbf{c}$ Distribution of $\mathrm{m}^{6} \mathrm{~A}$ peaks along transcripts in wild type and $Y_{t h d f 2^{-1-}}$. d Scatter plot showing $\mathrm{m}^{6} \mathrm{~A}$ peaks with increased (red) or decreased (green) levels. e Representative $\mathrm{m}^{6} \mathrm{~A}$ distribution along Nrp2 transcript. Enrichment coverage of $\mathrm{m}^{6} \mathrm{~A}$ and input are displayed as red and blue, respectively. Grey lines define coding sequence (CDS) borders

non-methylated actin was used as negative control (Fig. 6a). RT-qPCR showed that target genes were markedly enriched in Ythdf $2^{-/-}$neurospheres compared with the wild type (Fig. 6b). Next, we analyzed whether these $\mathrm{m}^{6} \mathrm{~A}$-enriched genes are real Ythdf2 targets by RNA IP (RIP) analysis. We confirmed that the Ythdf2 antibody was applicable to IP (Additional file 1: Figure S9). Compared with Ythdf2 $2^{-/}$, Nrp2 mRNA and other candidates were enriched by Ythdf2 protein in the wild type, which was verified by qPCR (Fig. 6c). To examine whether increased gene expression was due to loss of Ythdf2-mediated RNA decay, we measured the mRNA life time of these candidate genes by inhibition of transcription with actinomycin D in wild-type and Ythdf $2^{-/-}$neurospheres. After actinomycin D treatment, mRNA levels of Nrp2 and the other candidate genes in wild type declined more rapidly than in Ythdf2 $2^{-/-}$neurospheres (Fig. 6d and Additional file 1: Figure S10). Thus, the increased levels of $\mathrm{m}^{6} \mathrm{~A}$ modified mRNA transcripts in the absence of Ythdf2 were caused by delayed mRNA clearance, which might contribute to the defects in neurogenesis.

\section{Conclusions}

Ythdf2 is essential for oocyte maturation and early zygotic development in zebrafish and mouse [13, 25]. Ythdf2 was recently reported to be required for oocyte competence through the post-transcriptional regulation of the maternal transcriptome and homozygous $Y$ thdf $2^{-/-}$mice were reported to be partially permissive at weaning, with approximately $80 \%$ loss of homozygous $Y$ thd $f 2^{-/-}$mice in inbred C57BL/6 mice [25]. The targeting of the Ythdf2 locus described here caused a complete loss of 



Fig. 6 Ythdf2 is required for regulating mRNA decay of $m^{6} \mathrm{~A}$-modified neuron-related gene targets. a $\mathrm{m}^{6} \mathrm{~A}$ enrichment of target sites in gene candidates, verified by $\mathrm{m}^{6} \mathrm{~A}$ IP combined with RT-qPCR. Non- $\mathrm{m}^{6} \mathrm{~A}$-modified gene Actin was used as negative control. b Gene expression of gene candidates, verified by RT-qPCR with input RNA. Non-changed gene Actin was used as negative control. c Ythdf2 binding levels of gene candidates, verified by Ythdf2 RIP combined with RT-qPCR. Non- ${ }^{6}$ A-modified gene Actin was used as negative control. $\mathbf{d}$ Representative mRNA profile of Nrp2 at $0-, 2-$, and 4-h time points after actinomycin D $(5 \mu \mathrm{g} / \mathrm{ml})$ treatment (h.p.t.) in wild type and Ythdf $^{-/-}$. Error bars represent mean \pm standard deviation, $n=2$ biological replicates. ${ }^{*} P<0.05,{ }^{* *} P<0.01,{ }^{* * *} P<0.001$, Student's $t$-test 
homozygous Ythdf2 ${ }^{-/-}$mice in our inbred C57BL/6 background and the majority of $Y$ thdf $2^{-/-}$embryos died at late embryonic stages (Fig. 1). Of note, intercrossing Ythdf $2^{+/-}$mice results in constantly smaller litter size than from wild type matings (Fig. 1e), indicating an essential role of Ythdf2 in early embryo development. Here we reveal a crucial role of $\mathrm{m}^{6} \mathrm{~A}$ in mRNA and its binding protein Ythdf2 in neural development at embryonic developmental stages. The mammalian nervous system arises from the ectoderm, with both neurons and glial cells (astrocytes and oligodendrocytes) generated from NSCs in a precisely regulated spatiotemporal order [34]. We propose that erroneous recognition and degradation of $\mathrm{m}^{6} \mathrm{~A}$-containing mRNA at this stage leads to the dysregulation of neural development.

The $\mathrm{m}^{6} \mathrm{~A}$ level in mRNAs is higher in brain than in other studied mouse organs, indicating a crucial role during normal brain development [11]. Recent studies found $\mathrm{m}^{6} \mathrm{~A}$-modifying enzymes Mettl3, Alkbh5, and Fto to be involved in regulating progression of glioblastoma, indicating that $\mathrm{m}^{6} \mathrm{~A}$ epitranscriptomic regulation plays roles in the nervous system. Very recently, Yoon et al. [27] used a methyltransferase Mettl3-Mettl14 complex knockout to demonstrate that $\mathrm{m}^{6} \mathrm{~A}$ depletion extends cortical neurogenesis by protracting cell cycle progression of NPCs. In this study, we demonstrate the severe impact of Ythdf2 deletion on corticogenesis, neurogenesis, and gliogenesis. During early neural development, the decreased thickness of cortex is attributed to the dramatically thinner $\mathrm{CP}$ and SVZ layers, composed of neurons $\left(\mathrm{Dcx}^{+}\right)$and basal progenitor cells $\left(\mathrm{Tbr} 2^{+}\right)$, respectively (Figs. 2c and 3a). Multiple factors are supposed to contribute to this. First, consistent with the documented function of $\mathrm{m}^{6} \mathrm{~A}$ in proliferation of NPCs [27], our in vivo and in vitro evidence reveals that the proliferation capability of the NSPCs is severely compromised in Ythdf2 ${ }^{-/-}$embryonic cortex NSPCs. Further, apical progenitors symmetrically divide into more apical progenitors to expand the stem cell/ progenitor VZ pool [34]. No significant change in the thickness of the VZ layer in Ythdf2 ${ }^{-/-}$embryos suggests that the symmetric division of apical progenitors is not disturbed. However, apical mitosis is significantly decreased in Ythdf2 $2^{-/}$embryos. Apical progenitor cells can give rise to basal progenitor cells and neurons by asymmetric division $[40,41]$. The switch between symmetric and asymmetric cell division of $\mathrm{Ythdf}^{-/-}$ neural progenitor cells, which determines self-renewal or differentiation, may be disturbed. It is worth mentioning that it is not confirmed that the neural defects observed contribute to embryonic lethality. So it will be interesting to address this relationship by generating neural-specific Ythdf2 knockout mice. Second, the NSPCs derived from Ythdf $2^{-/}$embryo brains generated similar numbers of neurons as wild type in vitro (Fig. 4c and Additional file 1: Figure S2c). However, morphological analysis demonstrated abnormal neurite outgrowth of Ythdf $2^{-/-}$neurons that are more vulnerable to stress and fail to recover from neurite degeneration (Fig. 4e, $f$ and Additional file 1: Figure S3). Proper neurite outgrowth and branching is pivotal for establishing neuronal circuits which facilitate nervous system function [42]. Interestingly, RNA-seq analysis shows that differentially expressed genes (DEGs) relate to functions such as axon regulation, synapse assembly, and neuron differentiation (Additional file 1: Figure S4). Among them, genes such as Ddr2, Rnf135, Flrt2, Hlf, Nrp2, Nrxn3, and Ptprd have both up-regulated mRNA and $\mathrm{m}^{6} \mathrm{~A}$ levels (Fig. 6a, b). Ythdf2-mediated mRNA decay affects the translation efficiency and lifetime of $\mathrm{m}^{6} \mathrm{~A}$-modified mRNA targets [7]. By recruiting the Ccr4-not deadenylase complex, Ythdf2 initiates the degradation of its mRNA targets at specialized decay sites [43]. The RIP combined RT-qPCR and mRNA life-time assays display that mRNA levels of these genes are stabilized due to the complete absence of Ythdf2 in Ythdf2 ${ }^{-/-}$NSPCs (Fig. 6c, d and Additional file 1: Figure S10). Delayed mRNA degradation causes the retention of $\mathrm{m}^{6} \mathrm{~A}$ modified transcripts in Ythdf2 $2^{-/-}$neurospheres, leading to increased $\mathrm{m}^{6} \mathrm{~A}$ enrichment.

Last but not least, while homozygous $Y$ thdf2 $2^{-/-}$is embryonic lethal, heterozygous $Y$ thdf $2^{+/-}$is unexpectedly only partially lethal, with $30 \%$ of the surviving Ythdf2 $2^{+-}$ mice having eye defects (Additional file 1: Figure S1b), which may reflect haploid insufficiency of Ythdf2 and a malfunctioning nervous system. Furthermore, $\mathrm{m}^{6} \mathrm{~A}$ is highly enriched in mouse brain, and the level is dramatically increased with postnatal aging [11]. Taken together, we propose that $\mathrm{m}^{6} \mathrm{~A}$ and Ythdf2 have a pivotal function in brain not only during embryonic neural development but also in postnatal life. Thus, functions of $\mathrm{m}^{6} \mathrm{~A}$ and Ythdf2 on postnatal nervous system development merits further investigations.

During revision of this manuscript, two studies relating to the role of $\mathrm{m}^{6} \mathrm{~A}$ in the adult mammalian nervous system were reported. One study found that either the $\mathrm{m}^{6} \mathrm{~A}$ methyltransferase Mettl14 or the $\mathrm{m}^{6} \mathrm{~A}$-binding protein Ythdf1 regulate functional axon regeneration in the peripheral nervous system in vivo by modulating injury-induced protein translation [44]. In another study it was discovered that $\mathrm{m}^{6} \mathrm{~A}$ in $\mathrm{mRNA}$ regulates histone modification in part by destabilizing transcripts that encode histone-modifying enzymes, which might be a previously unknown mechanism of gene regulation in mammalian cells [45].

In summary, our study demonstrates a pivotal function of Ythdf2-mediated $\mathrm{m}^{6} \mathrm{~A}$ epitranscriptomic regulation in cortical neurogenesis during embryonic neural development, via regulating RNA degradation of 
$\mathrm{m}^{6} \mathrm{~A}$-tagged genes associated with neural development and differentiation.

\section{Methods}

Generation of conditionally Ythdf2 gene-targeted mice

The Ythdf2 conditional knockout mouse model (mYthdf2CKO) was generated as described in Additional file 1: Figure S1a by Applied Systemcell Inc. (CA, USA) using CRISPR-Cas9 technology. A cocktail of active guide RNA molecules (gRNAs), two single-stranded oligo donor nucleotides (ssODNs) and qualified Cas-9 mRNA was microinjected into the cytoplasm of C57BL/6 embryos. Two LoxP sites were inserted, flanking the upstream of 5' UTR and intron 1 regions, resulting in loss and changes in size of PCR products. Ythdf $2^{f l / f l}$ mice were genotyped and further sequenced for the LoxP cassettes at the designated locations. Potential Ythdf2-CKO mice were generated by crossing Ythdf $2^{f l / f l}$ mice with Cre_Del_GT_07 mice from the Norwegian Transgenic Center (NTS, Oslo, Norway).

For Ythdf2 genotyping, ear-clip samples were lysed in alkaline lysis reagent (25 mM NaOH, 0.2 mM EDTA, pH 12) at $95{ }^{\circ} \mathrm{C}$ for $30 \mathrm{~min}$, followed by adding neutralization reagent (40 mM Tris- $\mathrm{HCl}, \mathrm{pH} 5)$. PCR conditions for wild type and knockouts: $95^{\circ} \mathrm{C}, 2 \mathrm{~min}, 1$ cycle; $95^{\circ} \mathrm{C}, 30 \mathrm{~s} ; 60^{\circ} \mathrm{C}$, $30 \mathrm{~s} ; 72{ }^{\circ} \mathrm{C}, 1 \mathrm{~min} ; 35$ cycles. The PCR products were described in Additional file 1: Figure S1b. Primers for genotyping were as follows: wild-type allele (WT), 5'-TAC GGGTGAGGTGTCTTTTTCTT-3', 5'-GAAAGAGAGG AAACGAGGAAG-3'; targeted allele (KO), 5'-GGCTC TCCCTTCCCGAGAT-3', 5'-GCTTTTGTCCCTGACAC TCG-3'.

\section{Antibodies}

The following antibodies were used at the appropriate dilutions: mouse anti-Map2 (M4403, Sigma), rabbit anti-Gfap (Z0334, DAKO), mouse anti-Tuj1 (MAB1195, R\&D Systems), rabbit anti-s100- $\beta$ (ab52642, Abcam), rabbit anti-Dcx (ab18723, Abcam), rabbit anti-Tbr2 (ab23345, Abcam), rabbit anti-phospho-Histone H3 (PHH3; Ser10; 06-570, Millipore), mouse anti-Sox2 (ab79351, Abcam), mouse antiNestin (MAB353, Millipore), rabbit anti-Ythdf2 (RN123PW, MBL), mouse anti-anti- $\beta$-actin (A1978, Sigma).

\section{RT-qPCR analysis}

The total RNA was isolated using TRIzol LS Reagent (Life Technologies, 10,296-010). Normally, $1 \mu \mathrm{g}$ total RNA was used for reverse transcription using High-Capacity cDNA Reverse Transcription Kit (ThermoFisher, 4,368,814). The quantitative PCR reactions were carried out with Power SYBR Green PCR Master Mix (Life Technologies, $4,368,708)$ on a StepOnePlus ${ }^{\text {TM }}$ Real-Time PCR System instrument (Applied Biosystems). Primers used in this study were as follows.

\begin{tabular}{ll}
\hline Primers & \\
Ddr2 & Forward, TTGGCCACCCAAACAATCCA \\
Mob3b & Reverse, AGACCCCTCTGGTCACCAAC \\
& Forward, GAAAGCGATCCTGACTTCCAG \\
Rnf135 & Reverse, GCTAGCAGCACTTAGAGGGT \\
& Forward, ACTGGGAAGTGGACACTAGG \\
Speg & Reverse, CCAGGAGTCCATAGTCCTTCC \\
& Forward, CTAGTGGTGCGGGCAAATCT \\
Flrt2 & Reverse, CCTGGTAGCGGGAATGGT \\
& Forward, GACTGCCACATCCCCAACAA \\
HIf & Reverse, CACCTITCTAACGCTGGACCT \\
& Forward, CTGAAGGAGAACCAGATCGCA \\
Nrp2 & Reverse, TTCTTGCATTGCCCAGCTC \\
& Forward, CCCTITGGAAACTGAATGCCA \\
Nrxn3 & Reverse, GATCCCCTTCACAGCTGCAT \\
& Forward, ACGTATGGGCTCCATTCCT \\
Ptprd & Reverse, TTCTTGAGGCTCCCGTGAG \\
Soat1 & Forward, TGAGCCATACAGGGCACTTG \\
& Reverse, GCCTCCTAAGTCAGGATTCTTGT \\
Actin & Forward, GTGCAAGGGTGAGCCTATGT \\
& Reverse, GTGTGAGCAACTTGTACGGC \\
& Forward, TTCTITGCAGCTCCTTCGT \\
& Reverse, ATGGAGGGAATACAGCCC \\
\hline
\end{tabular}

\section{Western blotting}

Total protein lysate was extracted with RIPA buffer (20 mM Tris- $\mathrm{HCl}, \mathrm{pH} 7.4,20 \%$ glycerol, 0.5\% NP40, $1 \mathrm{mM} \mathrm{MgCl}_{2}, 150 \mathrm{mM} \mathrm{NaCl}, 1 \mathrm{mM}$ EDTA, $1 \mathrm{mM}$ EGTA). Protein concentrations were measured using the Bradford Assay, and 50-100 $\mu \mathrm{g}$ protein extracts were subjected to SDS-PAGE. Then proteins were transferred to a nitrocellulose membrane, blocked with 5\% non-fat milk and incubated with first antibodies for $1 \mathrm{~h}$ at room temperature. After incubation with secondary antibody against mouse $(1: 10,000)$ or rabbit $(1: 10,000)$ for $1 \mathrm{~h}$ at room temperature, the membrane was visualized with an ECL Western Blotting Detection Kit (32,106, Thermo).

\section{Immunohistochemistry and immunofluorescence}

For immunohistochemistry, embryonic brain tissues were dissected in cold PBS and fixed in $4 \%$ PFA at $4{ }^{\circ} \mathrm{C}$ for $48 \mathrm{~h}$. Slides ( $4 \mu \mathrm{m}$ thick) were sectioned by microtome (HM355s, Thermo Scientific) and deparaffinized and cleared in Clear-Rite ${ }^{\mathrm{Tm}} 3$ (6901TS, Thermo) followed by rehydration in an EtOH gradient. After antigen retrieval in citrate buffer ( $\mathrm{pH}$ 6.4), the slides were blocked with blocking buffer (5\% goat gut, $5 \%$ BSA, $0.1 \%$ tween20, $0.5 \%$ Triton $\mathrm{X}-100$ ) for $1 \mathrm{~h}$, and incubated with primary antibodies overnight at $4{ }^{\circ} \mathrm{C}$. Secondary antibodies were applied at room temperature for $1 \mathrm{~h}$. For immunofluorescence, cultured cells were fixed with $4 \%$ paraformaldehyde (PFA), permeabilized with $0.1 \%$ Triton $\mathrm{X}-100$, and stained with primary antibodies and secondary 
antibodies. Nuclei were visualized with mounting medium with DAPI (BioNordika, H-1200). Images were taken with a Leica SP8 confocal microscope equipped with a $\times 40$ oil immersion lens.

\section{H\&E staining}

Tissue slides were stained in haematoxylin (Richard-Allen Scientific, 12,687,756) and eosin (Nerliens Meszansky, 161,170) after dehydration and rehydration, followed by differentiation in acetic acid in $100 \%$ ethanol at 1:50,000 dilution for $5 \mathrm{~s}$. Then, the sections were dehydrated in ascending series of ethanol, treated with xylene, and coverslipped using Cytoseal XYL xylene-based mounting medium (8312-4, Thermo). Images were taken with a Zeiss AxioPlan 2 microscope system.

\section{TUNEL assay}

Cells were grown on coverslips, fixed on ice with $4 \%$ PFA for $10 \mathrm{~min}$, and permeabilized with $0.2 \%$ Triton $\mathrm{X}-100$ in PBS-Tween for $30 \mathrm{~min}$ on ice. After incubating in $3 \% \mathrm{H}_{2} \mathrm{O}_{2}$ in $\mathrm{PBS}$ for $10 \mathrm{~min}$, slides were rinsed twice with PBST. Slides were incubated with $50 \mu \mathrm{l}$ TUNEL reaction mixture for $60 \mathrm{~min}$ at $37{ }^{\circ} \mathrm{C}$. Nuclei were visualized with mounting medium with DAPI (BioNordika, H-1200). Images were taken with a Leica SP8 confocal microscope equipped with a $\times 40$ oil immersion lens.

\section{Neurosphere proliferation and differentiation}

Neurospheres derived from E14.5 embryonic fore brains were cultured with DMEM/F12 (GIBCO) supplemented with $20 \mathrm{ng} / \mathrm{ml}$ EGF (R\&D Systems, 236-EG-200), $10 \mathrm{ng} / \mathrm{ml}$ bFGF (R\&D Systems, 234-FSE 025), N2 supplement (Life, 17,502-048), and B27 supplement without vitamin A (Thermo, 12,587,010). Under the proliferating condition, cells were grow as free-floating neurospheres. For secondary neurosphere formation, cells in primary neurospheres were trypsinized with TrypLE ${ }^{\mathrm{TM}}$ Express Enzyme (Gibco, 12,604,021) combined with DNaseI (Thermo, 18,047,019), dissociated mechanically by pipetting onto a six-well plate at $5 \times 10^{5}$ cells per well. For the neurosphere differentiation assay, a set of neurospheres were trypsinized to obtain a suspension of dissociated cells. These cells were then plated in tissue culture plates pre-coated with poly-L-lysine (Sigma, P6516). Cells were cultured in differentiation medium (minus EGF and bFGF) and collected at different time points.

\section{Proliferation assay}

Dissociated single NSPCs were seeded at a density of $1.0 \times 10^{4}$ per well in 96-well plates. The proliferation rates were measured at 24,72 , and $120 \mathrm{~h}$ with PrestoBlue Cell Viability reagent (A13262, ThermoFisher Scientific) as instructed.

\section{Ythdf2 RIP}

Ythdf2 RIP was carried out with a modified procedure [46]. Briefly, $1 \times 10^{7}$ collected NSPCs were lysed in NETN buffer (20 mM Tris-Cl, pH 8.0; $100 \mathrm{mM} \mathrm{NaCl}$, $1 \mathrm{mM}$ EDTA, 0.5\% NP-40, freshly added protease inhibitor cocktail and RNasin) for 20 min on ice. After centrifugation, the supernatant containing the RNA-protein complex was incubated with $5 \mu \mathrm{g}$ Ythdf2 antibody (RN123PW, MBL) for $2 \mathrm{~h}$ at $4{ }^{\circ} \mathrm{C}$. Then $30 \mu$ Dynabeads $\mathrm{G}$ beads were added and rotated for $2 \mathrm{~h}$, at $4{ }^{\circ} \mathrm{C}$. Beads were collected with a magnetic stand and washed with NETN buffer four times. The RNA-protein complex was eluted by incubating with NETN buffer with $0.1 \%$ SDS and $30 \mu \mathrm{g}$ proteinase $\mathrm{K}$ at $50{ }^{\circ} \mathrm{C}$ for $30 \mathrm{~min}$. RNAs were further purified with RNA Clean and Concentrator-5 (Zymo).

\section{LC-MS/MS}

Purified mRNA was digested by nuclease P1 (2 U, Wako) in $25 \mu \mathrm{l}$ of buffer containing $10 \mathrm{mM}$ of $\mathrm{NH}_{4} \mathrm{OAc}$ $(\mathrm{pH} 5.3)$ at $42{ }^{\circ} \mathrm{C}$ for $2 \mathrm{~h}$, followed by the addition of $\mathrm{NH}_{4} \mathrm{HCO}_{3}(1 \mathrm{M}, 3 \mu \mathrm{l}$, freshly made) and alkaline phosphatase $(0.5 \mathrm{U})$. After an additional incubation at $37{ }^{\circ} \mathrm{C}$ for $2 \mathrm{~h}$, the sample was diluted to $50 \mu \mathrm{l}$ and filtered ( 0 . $22 \mu \mathrm{m}$ pore size, $4 \mathrm{~mm}$ diameter, Millipore), and $5 \mu \mathrm{l}$ of the solution was subjected to LC-MS/MS. Nucleosides were separated by reverse-phase ultra-performance liquid chromatography on a C18 column with on-line mass spectrometry detection using an Agilent 6410 QQQ triple-quadrupole LC mass spectrometer in positive electrospray ionization mode. The nucleosides were quantified using the nucleoside to base ion mass transitions of 282 to $150\left(\mathrm{~m}^{6} \mathrm{~A}\right)$ and 268 to 136 (A). Quantification was performed in comparison with the standard curve obtained from pure nucleoside standards running on the same batch of samples. The ratio of $\mathrm{m}^{6} \mathrm{~A}$ to A was calculated based on the calibrated concentrations.

\section{mRNA isolation and $\mathrm{m}^{6} \mathrm{~A}-\mathrm{RIP}$}

NSPCs $\left(1 \times 10^{7}\right)$ dissociated from neurospheres were collected for total RNA isolation with Direct-zol RNA miniprep plus with TRI Reagent (Zymo research, R2073) and DNase I digestion following the manufacturer's instructions. We applied $1 \mathrm{mg}$ total RNA for further mRNA purification with a Dynabeads mRNA DIRECT ${ }^{\mathrm{Tm}}$ purification kit (Thermo, 61,011) for two rounds. The mRNA quality was checked using a 2100 Bioanalyzer instrument with an Agilent RNA 6000 Nano kit (5067-1511).

RNA fragmentation $(1 \mu \mathrm{g})$ was performed by sonication at $10 \mathrm{ng} / \mu \mathrm{l}$ in $100 \mu \mathrm{l}$ RNase-free water with Bioruptor Pico (Diagenode) with 30 cycles of $30 \mathrm{~s}$ on followed by $30 \mathrm{~s}$ off; $5 \%$ of the fragmented RNA was saved as input. $\mathrm{m}^{6} \mathrm{~A}$ IP was performed with an EpiMark $N^{6}$-Methyladenosine Enrichment Kit (NEB, E1610S) 
following the kit manual adapted for the KingFisher ${ }^{\mathrm{Tx}}$ Duo Prime Purification System. In detail, $1 \mu \mathrm{l}$ N6methyladenosine antibody from the kit and $25 \mu \mathrm{l}$ Protein $G$ beads (NEB \#S1430) were used for each affinity pull down. After incubating with RNA, the beads were washed with $200 \mu \mathrm{l}$ low salt reaction buffer twice, and then $200 \mu \mathrm{l}$ high salt reaction buffer twice. RNA that was pulled down (IP) was eluted with $50 \mu \mathrm{l}$ RLT buffer twice, and recovered by RNA Clean and Concentrator- 5 (Zymo). Both input and IP were subjected to RNA library preparation with Truseq Stranded mRNA Library Prep Kit with the RFP incubation step shorten from 8 min to $20 \mathrm{~s}$. Sequencing was carried out on Illumina HiSeq 4000 according to the manufacturer's instructions.

\section{Sequencing data analysis}

The sequencing data were mapped to mouse genome version mm10 downloaded from UCSC. Data analysis was carried out as previously described. Briefly, reads were aligned to mm10 using TopHat v2.0.142. For input analysis (RNA-seq), RPKM were calculated by Cuffnorm3. For $\mathrm{m}^{6} \mathrm{~A}$ peak calling, the longest isoform was used if multiple isoforms were detected. Aligned reads were extended to 100 nucleotides (average fragment size) and converted from genome-based coordinates to isoformbased coordinates to eliminate interference from introns in peak calling. The longest isoform of each mouse gene was scanned using a 100-nucleotide sliding window with 10-nucleotide steps. To reduce bias from potentially inaccurate gene structure annotation and the arbitrary use of the longest isoform, windows with read counts less than $1 / 20$ of the top window in both $\mathrm{m}^{6} \mathrm{~A}$ IP and input samples were excluded. For each gene, the read count in each window was normalized by the median count of all windows of that gene. The window was called positive if $\mathrm{FDR}<1 \%$ and $\log 2$ (enrichment score) $\geq 1$. Overlapping positive windows were merged. The following four numbers were calculated to obtain the enrichment score of each peak (or window): read count of the IP sample in the current peak/window (a); median read count of the IP sample in all 100-nucleotide windows on the current mRNA (b); read count of the input sample in the current peak/window (c); and median read count of the input sample in all 100-nucleotident windows on the current mRNA (d). The enrichment score of each window was calculated as $(a \times d) /(b \times c)$. Common peaks shared in the triplicates of a sample were kept with the peak annotation from replicate 1 .

For motif analysis, consensus motif was determined by using HOMER4. For GO analysis, differentially expressed genes or $\mathrm{m}^{6} \mathrm{~A}$-modified genes were uploaded to DAVID (http://david.abcc.ncifcrf.gov/). The GO terms were ranked and presented according to $-\log 2(P$ value).
mRNA life-time assay

Wild-type and $Y$ thdf2 $2^{-/-}$neurospheres were trypsinized with TrypLE ${ }^{\mathrm{m}}$ Express Enzyme (Thermo, 12,605,010). The dissociated cells were seeded into plates coated with PDL (Millipore, A-003-E) and laminin (R\&D Systems, 3446-005-01). After 12-h culturing, cells were treated with $5 \mu \mathrm{g} / \mathrm{ml}$ actinomycin D (Sigma, A9415) for 2 and $4 \mathrm{~h}$, while cells without treatment were used as $0 \mathrm{~h}$. Cells were collected at designated time points and total RNA was extracted for reverse transcription and qPCR.

\section{Statistical analysis}

All statistical analyses were performed with GraphPad Prism 5. Student's $t$-test was adapted and data are shown as mean \pm standard deviation. $P$ value is used for significance.

\section{Additional file}

Additional file 1: Figures S1-S10. This document contains additional supporting evidence for this study presented in the form of supplemental figures. (PDF $1160 \mathrm{~kb}$ )

\section{Acknowledgments}

We thank the animal facility at Oslo University Hospital for mouse handling.

\section{Funding}

This work was funded by the Norwegian Cancer Society (to A.K. and M.B.), the Norwegian Research council, the Health Authority South East, and National Institute of Health (RM1 HG008935 to C.H.). C.H. is an investigator of the Howard Hughes Medical Institute.

\section{Availability of data and materials}

High-throughput sequencing data have been deposited in the Gene Expression Omnibus database under accession number GSE104867 [47]. All the other data generated or analyzed during this study are included in the article and additional files.

\section{Authors' contributions}

$\mathrm{AK}, \mathrm{XZ}, \mathrm{MML}$ and $\mathrm{MB}$ conceived the project, designed the experiments, and wrote the manuscript. MML and $X Z$ performed the experiments with the help of WW and SPP; HLS and CH provided sequencing and mass spectrometry analyses; XZ designed and QFP performed the bioinformatics analysis. AK, XZ and $M M L$ drafted the manuscript with substantial input from all co-authors. All authors approved the paper.

\section{Ethics approval and consent to participate}

All mouse experiments were approved by the Norwegian Animal Research Authority by Norwegian Food Safety Authority and done in accordance with institutional guidelines at the Centre for Comparative Medicine at Oslo University Hospital. Animal work was conducted in accordance with the rules and regulations of the Federation of European Laboratory Animal Science Association's (FELASA).

\section{Competing interests}

C. H. is a scientific founder of Accent Therapeutics, Inc. The other authors declare that they have no competing interests.

\section{Publisher's Note}

Springer Nature remains neutral with regard to jurisdictional claims in published maps and institutional affiliations. 


\section{Author details}

'Department of Microbiology, Oslo University Hospital, Rikshospitalet, NO-0027 Oslo, Norway. ${ }^{2}$ Department of Molecular Medicine, Institute of Basic Medical Sciences, University of Oslo, NO-0317 Oslo, Norway. ${ }^{3}$ Department of Clinical and Molecular Medicine, Norwegian University of Science and Technology (NTNU), Trondheim, Norway. ${ }^{4}$ Department of Chemistry, Institute for Biophysical Dynamics, The University of Chicago, 929 East 57th Street, Chicago, IL 60637, USA. ${ }^{5}$ Howard Hughes Medical Institute, The University of Chicago, 929 East 57th Street, Chicago, IL 60637, USA. ${ }^{6}$ Department of Computational Biology, St. Jude Children's Hospital, Memphis, TN 38105, USA.

Received: 3 December 2017 Accepted: 26 April 2018 Published online: 31 May 2018

\section{References}

1. Roundtree IA, Evans ME, Pan T, He C. Dynamic RNA modifications in gene expression regulation. Cell. 2017;169:1187-200.

2. Saletore Y, Meyer K, Korlach J, Vilfan ID, Jaffrey S, Mason CE. The birth of the Epitranscriptome: deciphering the function of RNA modifications. Genome Biol. 2012;13:175

3. Jia GF, Fu Y, Zhao X, Dai Q, Zheng GQ, Yang Y, et al. N6-Methyladenosine in nuclear RNA is a major substrate of the obesity-associated FTO. Nat Chem Biol. 2011:7:885-7.

4. Desrosiers RC, Friderici KH, Rottman FM. Characterization of Novikoff hepatoma mRNA methylation and heterogeneity in the methylated $5^{\prime}$ terminus. Biochemistry. 1975;14:4367-74.

5. Schwartz S, Mumbach MR, Jovanovic M, Wang T, Maciag K, Bushkin GG, et al. Perturbation of m6A writers reveals two distinct classes of mRNA methylation at internal and 5' sites. Cell Rep. 2014;8:284-96.

6. Zheng G, Dahl JA, Niu Y, Fedorcsak P, Huang CM, Li CJ, et al. ALKBH5 is a mammalian RNA demethylase that impacts RNA metabolism and mouse fertility. Mol Cell. 2013:49:18-29.

7. Wang X, Lu Z, Gomez A, Hon GC, Yue Y, Han D, et al. N6-methyladenosinedependent regulation of messenger RNA stability. Nature. 2014;505:117-20.

8. Xiao W, Adhikari S, Dahal U, Chen YS, Hao YJ, Sun BF, et al. Nuclear m(6)A Reader YTHDC1 Regulates mRNA Splicing. Mol Cell. 2016:61:507-19.

9. Hsu PJ, Zhu Y, Ma H, Guo Y, Shi X, Liu Y, et al. Ythdc2 is an N6methyladenosine binding protein that regulates mammalian spermatogenesis. Cell Res. 2017;27:1115-27.

10. Dominissini D, Moshitch-Moshkovitz S, Schwartz S, Salmon-Divon M, Ungar $L$, Osenberg $S$, et al. Topology of the human and mouse m6A RNA methylomes revealed by m6A-seq. Nature. 2012;485:201-6.

11. Meyer KD, Saletore $Y$, Zumbo $P$, Elemento $O$, Mason $C E$, Jaffrey $S R$. Comprehensive analysis of mRNA methylation reveals enrichment in 3' UTRs and near stop codons. Cell. 2012:149:1635-46.

12. Shah JC, Clancy MJ. IME4, a gene that mediates MAT and nutritional control of meiosis in Saccharomyces cerevisiae. Mol Cell Biol. 1992:12: 1078-86.

13. Zhao BS, Wang X, Beadell AV, Lu Z, Shi H, Kuuspalu A, et al. m6Adependent maternal mRNA clearance facilitates zebrafish maternal-tozygotic transition. Nature. 2017;542:475-8.

14. Zhao $X$, Yang $Y$, Sun BF, Shi $Y$, Yang $X$, Xiao W, et al. FTO-dependent demethylation of N6-methyladenosine regulates mRNA splicing and is required for adipogenesis. Cell Res. 2014:24:1403-19.

15. Boissel S, Reish O, Proulx K, Kawagoe-Takaki H, Sedgwick B, Yeo GS, et al. Loss-of-function mutation in the dioxygenase-encoding FTO gene causes severe growth retardation and multiple malformations. Am J Hum Genet. 2009;85:106-11.

16. Fustin JM, Doi M, Yamaguchi Y, Hida H, Nishimura S, Yoshida M, et al. RNAmethylation-dependent RNA processing controls the speed of the circadian clock. Cell. 2013;155:793-806

17. Wang Y, Li Y, Toth Jl, Petroski MD, Zhang Z, Zhao JC. N(6)-methyladenosine modification destabilizes developmental regulators in embryonic stem cells. Nat Cell Biol. 2014;16:191-8.

18. Batista PJ, Molinie B, Wang J, Qu K, Zhang J, Li L, et al. m(6)A RNA modification controls cell fate transition in mammalian embryonic stem cells. Cell Stem Cell. 2014;15:707-19.

19. Geula S, Moshitch-Moshkovitz S, Dominissini D, Mansour AA, Kol N, SalmonDivon $\mathrm{M}$, et al. m6A mRNA methylation facilitates resolution of naive pluripotency toward differentiation. Science. 2015;347(6225):1002-6.
20. Chen T, Hao YJ, Zhang Y, Li MM, Wang M, Han W, et al. m(6)A RNA methylation is regulated by microRNAs and promotes reprogramming to pluripotency. Cell Stem Cell. 2015;16:289-301.

21. Zhang $C$, Chen $Y$, Sun B, Wang L, Yang Y, Ma D, et al. m6A modulates haematopoietic stem and progenitor cell specification. Nature. 2017;549: 273-6.

22. Vu LP, Pickering BF, Cheng Y, Zaccara S, Nguyen D, Minuesa G, et al. The N6-methyladenosine (m6A)-forming enzyme METTL3 controls myeloid differentiation of normal hematopoietic and leukemia cells. Nat Med. 2017; 23:1369-76.

23. Shi H, Wang X, Lu Z, Zhao BS, Ma H, Hsu PJ, et al. YTHDF3 facilitates translation and decay of N6-methyladenosine-modified RNA. Cell Res. 2017;27:315-28.

24. Alarcon CR, Goodarzi H, Lee H, Liu X, Tavazoie S, Tavazoie SF. HNRNPA2B1 Is a Mediator of m(6)A-Dependent Nuclear RNA Processing Events. Cell. 2015; 162:1299-308.

25. Ivanova I, Much C, Di Giacomo M, Azzi C, Morgan M, Moreira PN, et al. The RNA m6A reader YTHDF2 is essential for the post-transcriptional regulation of the maternal transcriptome and oocyte competence. Mol Cell. 2017;67(6): 1059-67.

26. Lence T, Akhtar J, Bayer M, Schmid K, Spindler L, Ho CH, et al. m6A modulates neuronal functions and sex determination in Drosophila. Nature. 2016:540:242-7.

27. Yoon KJ, Ringeling FR, Vissers C, Jacob F, Pokrass M, Jimenez-Cyrus D, et al. Temporal control of mammalian cortical neurogenesis by $\mathrm{m} 6 \mathrm{~A}$ methylation. Cell. 2017;171(4):877-89.

28. Gotz M, Huttner WB. The cell biology of neurogenesis. Nat Rev Mol Cell Biol. 2005;6:777-88

29. Nordstrand LM, Svard J, Larsen E, Nilsen A, Ougland R, Furu K, et al. Mice lacking Alkbh1 display sex-ratio distortion and unilateral eye defects. PLoS One. 2010:5:e13827.

30. Castranio T, Mishina Y. Bmp2 is required for cephalic neural tube closure in the mouse. Dev Dyn. 2009:238:110-22.

31. Lizarraga SB, Margossian SP, Harris MH, Campagna DR, Han AP, Blevins S, et al. Cdk5rap2 regulates centrosome function and chromosome segregation in neuronal progenitors. Development. 2010;137:1907-17.

32. Englund C, Fink A, Lau C, Pham D, Daza RA, Bulfone A, et al. Pax6, Tbr2, and Tbr1 are expressed sequentially by radial glia, intermediate progenitor cells, and postmitotic neurons in developing neocortex. J Neurosci. 2005:25:247-51

33. Kang $W$, Wong LC, Shi SH, Hebert JM. The transition from radial glial to intermediate progenitor cell is inhibited by FGF signaling during corticogenesis. J Neurosci. 2009;29:14571-80.

34. Taverna E, Gotz M, Huttner WB. The cell biology of neurogenesis: toward an understanding of the development and evolution of the neocortex. Annu Rev Cell Dev Biol. 2014;30:465-502.

35. Chen $H$, Lin W, Zhang $Y$, Lin L, Chen J, Zeng $Y$, et al. IL-10 promotes neurite outgrowth and synapse formation in cultured cortical neurons after the oxygen-glucose deprivation via JAK1/STAT3 pathway. Sci Rep. 2016;6:30459.

36. Jomova K, Valko M. Advances in metal-induced oxidative stress and human disease. Toxicology. 2011;283:65-87.

37. Mishra D, Flora SJ. Differential oxidative stress and DNA damage in rat brain regions and blood following chronic arsenic exposure. Toxicol Ind Health. 2008;24:247-56

38. Justicia C, Gabriel C, Planas AM. Activation of the JAK/STAT pathway following transient focal cerebral ischemia: signaling through Jak1 and Stat3 in astrocytes. Glia. 2000;30:253-70.

39. Planas AM, Soriano MA, Berruezo M, Justicia C, Estrada A, Pitarch S, et al. Induction of Stat3, a signal transducer and transcription factor, in reactive microglia following transient focal cerebral ischaemia. Eur J Neurosci. 1996; 8:2612-8

40. Kriegstein A, Noctor S, Martinez-Cerdeno V. Patterns of neural stem and progenitor cell division may underlie evolutionary cortical expansion. Nat Rev Neurosci. 2006:7:883-90.

41. Merkle FT, Alvarez-Buylla A. Neural stem cells in mammalian development Curr Opin Cell Biol. 2006:18:704-9.

42. Conde C, Caceres A. Microtubule assembly, organization and dynamics in axons and dendrites. Nat Rev Neurosci. 2009:10:319-32.

43. Du H, Zhao Y, He J, Zhang Y, Xi H, Liu M, et al. YTHDF2 destabilizes m(6)Acontaining RNA through direct recruitment of the CCR4-NOT deadenylase complex. Nat Commun. 2016;7:12626. 
44. Weng YL, Wang X, An R, Cassin J, Vissers C, Liu Y, et al. Epitranscriptomic $m 6 \mathrm{~A}$ regulation of axon regeneration in the adult mammalian nervous system. Neuron. 2018;97:313-25.

45. Wang Y, Li Y, Yue M, Wang J, Kumar S, Wechsler-Reya RJ, et al. N6methyladenosine RNA modification regulates embryonic neural stem cell self-renewal through histone modifications. Nat Neurosci. 2018;21:195-206.

46. Peritz T, Zeng F, Kannanayakal TJ, Kilk K, Eiriksdottir E, Langel U, et al. Immunoprecipitation of mRNA-protein complexes. Nat Protoc. 2006;1:577-80.

47. Li M, Zhao X, Wang W, Shi H, Pan Q, Lu Z, et al. Ythdf2-mediated m6A mRNA clearance modulates neural development in mice. Data sets. NCBI GEO. 2018. https://www.ncbi.nlm.nih.gov/geo/query/acc.cgi?acc= GSE104867. Accessed 1 Jan 2018.

Ready to submit your research? Choose BMC and benefit from:

- fast, convenient online submission

- thorough peer review by experienced researchers in your field

- rapid publication on acceptance

- support for research data, including large and complex data types

- gold Open Access which fosters wider collaboration and increased citations

- maximum visibility for your research: over $100 \mathrm{M}$ website views per year 